\title{
A global error estimator for the uncertainty of a multi-channel spectral analysis
}

\author{
N.B. Roozen ${ }^{\mathrm{a}, \mathrm{b}, *}$, Q. Leclère $^{\mathrm{c}}$, C. Glorieux ${ }^{\mathrm{a}}$, M. Rychtárikováa \\ ${ }^{a}$ Katholieke Universiteit Leuven, Laboratory for Acoustics and Thermal Physics (ATF), \\ Department of Physics and Astronomy, Celestijnenlaan $200 D 3001$ Leuven, Belgium \\ ${ }^{b}$ Katholieke Universiteit Leuven, Department of Mechanical Engineering, Celestijnenlaan \\ 300 B, B-3001 Leuven, Belgium \\ ${ }^{c}$ Laboratoire Vibrations Acoustique, INSA-Lyon, 25 bis avenue Jean Capelle, F-69621 \\ Villeurbanne Cedex, France
}

\section{Abstract}

A global estimator of the uncertainty of the average frequency response function in multi-channel spectral analysis measurements is proposed. The proposed global estimator is a generalization of the random error estimator of the frequency response function magnitude of a single-input-single-output system. In principle, the signal-to-noise ratio (and thus the quality of the frequency response function estimation) is increasing with increasing number of averages $M$, according to $\sqrt{M}$. However, in the situation that, for practical reasons, there is a maximum imposed upon the total measurement time $T_{\max }$, it is clear that there is a trade-off between the number of averages $M$ and the record length $T[\mathrm{~s}]$ that is used to obtain an estimate of a single-averagefrequency-response-function. There is a choice between a few long records or many short records, with the requirement that, assuming zero overlap, the number of averages $M$ times the record length $T$ may not exceed the total available measurement time, i.e. $M \times T \leq T_{\max }$. In addition to the existence of such an optimum, a minimum record length is required as well which is related to the reverberation time of the system. The newly proposed global estimator is used to determine the optimal record length of a multichannel system, such that a minimum error of the average frequency response function is obtained. It is also shown by experimental results that indeed the

\footnotetext{
*Corresponding author

Email address: bert.roozen@kuleuven.be (N.B. Roozen)
} 
minimum allowable record length is related to the reverberation time of the system being measured.

Keywords: uncertainty, multi-channel, spectral analysis

PACS: 43.20.-f, 43.20.+g, 43.40.-r, 43.40.+s, 43.60.-c, 43.60.+d, 46.70.-p

\section{Introduction}

In this article a global estimator is proposed. For demonstration purposes the estimator is applied to the multi-channel spectral analysis of a vibrating building element. The measurements were carried out by means of a scanning laser Doppler vibrometer and were intended to determine the radiated sound power at low frequencies (as described in more detail in [1]). An interesting complicating factor in this experiment is that the building element is excited by a reverberant acoustic field, which exhibits a random characteristic in time. By looking for a minimum of the proposed global estimator, an optimum record length is sought, for a given reverberation time of the room. This is a classical problem, discussed by Piersol in 1978 [2], and later also by Jacobsen and Nielsen in 1987 [3], where it was concluded that the analysis record lengths should be at least as long as the reverberation time to avoid serious errors in the coherence estimate. The measurements also indeed demonstrate nicely that the minimum record length is required to be longer than the reverberation time of the system.

\section{Theory}

A multiple-channel-output system is considered as illustrated in Figure 1. A building element is excited by an acoustic field, as shown, resulting in a response being measured at different positions, $i=1 \ldots N$. An additional position $r$ is being probed simultaneously with each of the other positions, thus serving as a reference, allowing the responses at positions $i=1 \ldots N$ to be measured sequentially in time, retaining the phase information of the response at the individual positions (assuming the building element to respond stationary and time-independent). As we assume the exciting acoustic field to be caused by a single sound source (loudspeaker), we are dealing with a single-input-multiple-output system, for which a single reference point is sufficient. In case of a multiple-input-multiple-output system more reference points will be necessary. 


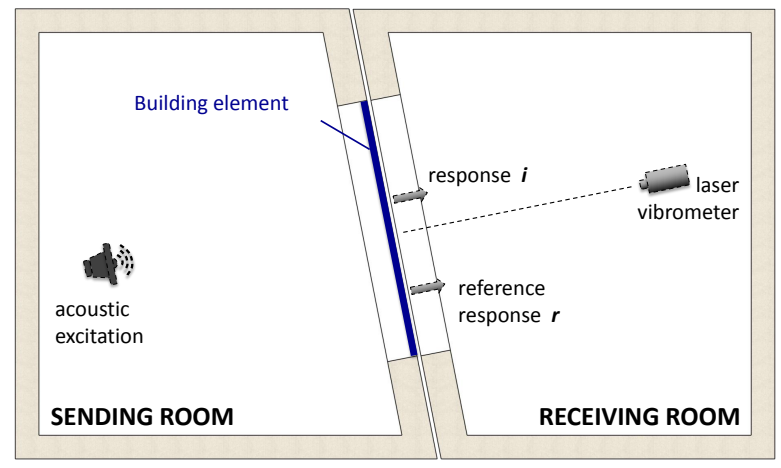

Figure 1: Test facility measurement set-up.

In the following the auto-spectra of the response of the structure at different positions is denoted by $S_{i i}$, with $i=1 \ldots N$, the auto-spectrum of the reference signal is denoted by $S_{r r}$, and the cross-spectra between the response of the structure at different positions and the reference signal are denoted by $S_{i r}$, with $i=1 \ldots N$. The frequency response function between the response of the structure at different positions and the one of some reference signal can then be estimated using the $H^{1}$-optimal estimate or the $H^{2}$-optimal estimate, respectively [4]:

$$
\begin{aligned}
& H_{i r}^{1}=\frac{S_{r i}}{S_{r r}} \\
& H_{i r}^{2}=\frac{S_{i i}}{S_{i r}}
\end{aligned}
$$

It is well known that measurement noise is better suppressed when averaging cross-spectra, compared to averaging auto-spectra $[4,5,6]$. Taking a cross-spectrum enhances common frequency components in both signals that have a consistent phase relationship. Uncorrelated signal components average to zero. Since auto-spectra are positive valued, summing them accumulates not only correlated signal components, but also uncorrelated ones.

In view of this, as the estimate of frequency response functions is based upon both cross-spectra and auto-spectra, it is best to choose the $H$-estimate with the least noisy autocorrelation factor, i.e. use $H^{1}$ in case less noise is expected on the reference auto-spectrum $S_{r r}$ and use $H^{2}$ if less noise is expected on the response auto-spectra $S_{i i}$. 


\subsection{Global coherence and global $\epsilon$}

A quantity that plays an important role in the statistical uncertainty of the averaged frequency response function of a single-input-single-output system is the coherence of the frequency response function, which is defined as [1]

$$
\gamma_{r i}=\frac{\left|S_{r i}\right|^{2}}{S_{r r} S_{i i}}
$$

Given the coherences $\gamma_{r i}$ related to each frequency response function $H_{r i}$, the normalized standard deviations due to random errors in single-inputsingle-output systems are given by [1]:

$$
\epsilon_{H_{r i}}=\frac{1}{\sqrt{2 M}} \sqrt{\frac{1-\gamma_{r i}}{\gamma_{r i}}}
$$

where $M$ is the number of averages.

As mentioned above, the goal of this paper is to introduce a global coherence function that considers the quality of all measurements simultaneously, as well as a global statistical uncertainty on the average frequency response function. As the coherence function $\gamma_{r i}$ as defined above can be seen as the ratio between the energy in the auto-spectrum of signal $i$ that is coherent with the reference signal, $\gamma_{r i} S_{i i}$, and the total energy in the the auto-spectrum of signal $i, S_{i i}$, we propose the following expression for the global coherence:

$$
\gamma^{\text {global }}=\frac{\left\langle\gamma_{r i} S_{i i}\right\rangle_{N}}{\left\langle S_{i i}\right\rangle_{N}}
$$

where $\langle. .\rangle_{N}$ denotes the average over all $N$ measurement points. The thus defined global coherence can be viewed as the ratio between the mean coherent auto-spectrum by the mean raw auto-spectrum.

In addition, we propose an estimate for the statistical uncertainty of the average frequency response function as follows:

$$
\epsilon_{H_{r i}}^{\text {global }}=\sqrt{\frac{\left\langle\epsilon_{H_{r i}}^{2}\left|H_{r i}^{2}\right|\right\rangle_{N}}{N\left\langle\left|H_{r i}\right|\right\rangle_{N}^{2}}}=\frac{\sqrt{\frac{\left\langle\epsilon_{H_{r i}}^{2}\left|H_{r i}\right|^{2}\right\rangle_{N}}{N}}}{\left\langle\left|H_{r i}\right|\right\rangle_{N}}
$$

Indeed, the variance of the modulus of the average frequency response function is given by 


$$
\mathbb{V}\left\{\left\langle\left|H_{r i}\right|\right\rangle_{N}\right\}=\frac{1}{N}\left\langle\mathbb{V}\left\{\left|H_{r i}\right|\right\}\right\rangle_{N}
$$

while

$$
\mathbb{V}\left\{\left|H_{r i}\right|\right\}=\left|H_{r i}\right|^{2} \epsilon_{H_{r i}}^{2}
$$

Hence, assuming a gaussian distribution, it can be stated with $95 \%$ certainty that the actual value of $\left\langle\left|H_{r i}\right|\right\rangle_{N}$ lies between $\left\langle\left|H_{r i}\right|\right\rangle_{N}-2 \epsilon_{H_{r i}}^{\text {global }}\left\langle\left|H_{r i}\right|\right\rangle_{N}$ and $\left\langle\left|H_{r i}\right|\right\rangle_{N}+2 \epsilon_{H_{r i}}^{\text {global }}\left\langle\left|H_{r i}\right|\right\rangle_{N}$.

Note that in [1] (section 8.2) a multiple coherence function was defined for a system with multiple inputs and a single output, which provides a measure of the linear dependence between a collection of inputs and an output, independent of the correlation among the inputs. However, to the best of our knowledge, a global coherence as defined above for a single input multiple output system, has not been proposed before. The same is true for the global error of the average of the frequency response function.

\subsection{The multi-pass estimator}

In the situation that the system being investigated responds stationary and time-independent, the frequency response functions $H_{r i}, i=1 \ldots N$, can be measured sequentially in time using a multi-pass measurement scheme, which puts less heavy demands on the measurement and data acquisition system in terms of the required number of channels. Assuming that a $H^{1}$-type of estimate of the frequency response functions is required, a fixed reference signal, here denoted as the signal measured at point $r$, should be used in order to retain the phase information at each point $i=1 \ldots N$, using Equation (1) for the estimation of the frequency response function:

$$
H_{r i}^{1}=\frac{S_{r i}}{S_{r r}}
$$

By estimating the frequency response function between this reference signal and the response of the structure at different positions, being measured sequentially in time, the phase between the response at the different measurement points and the reference can be determined, and thus also the phase between the individual responses.

A particularity of such a multi-pass measurement scheme is that the reference signal is acquired at each measurement pass, whilst all other signals are 
recorded during one pass only. Having $N$ measurement passes to determine the $N$ components of the frequency response functions $H_{r i}, i=1 \ldots N$, we thus have $N$ times an estimate of the reference auto-spectrum $S_{r r(i)}$, with $i=1 \ldots N$.

In this particular case, it is advantageous to estimate the auto-spectrum of the reference signal $S_{r r}$ as the averaged auto-spectrum of all sequentially in time determined auto-spectra $S_{r r(i)}$, with $i=1 \ldots N$, i.e.:

$$
\left\langle S_{r r}\right\rangle_{N}=\frac{1}{N} \sum_{i=1}^{N} S_{r r(i)}
$$

Taking the average auto-spectum of the input increases the signal-tonoise-ratio of the reference auto-spectrum with a factor $\sqrt{N}$. In combination with the frequency response functions for different locations $i$ calculated from respective auto-spectra and cross-spectra on the basis of records of limited duration, the averaged structural response function $X_{i}$ at each individual point $i$, can be estimated as:

$$
X_{i}=H_{r i}^{1} \sqrt{\left\langle S_{r r}\right\rangle_{N}}
$$

This method is called multi-pass acquisition [7].

Provided the noise contributions are uncorrelated, the error in the estimate of $\left\langle S_{r r}\right\rangle_{N}$ is a factor $\sqrt{N}$ smaller than the error in the estimate of $S_{r r}$, with obvious advantages for the accuracy of the $X_{i}$ estimate.

\section{Practical application}

In this section the multi-pass acquisition is used in a practical application, with the aim of illustrating its adequacy. In addition, the proposed

global estimator $\epsilon_{H_{r i}}^{\text {global }}$ is used to determine the optimal record length for the measurement, assuming a maximum allowable measurement time $T_{\max }$ of 100 [s], thus finding the best compromise between the number of averages $M$ and the record length $T[\mathrm{~s}]$. In addition, the minimum record length is being studied by the same estimator $\epsilon_{H_{r i}}^{g l o b a l}$. In the considered experiment, we determine the response of a light-weight double wall panel that is subjected to a random, diffuse acoustic field at one side. The building element is mounted in a transmission test facility as illustrated in Figure 1.

The transmission rooms are of identical shape, with a slightly inclined test partition (see Figure 1), and with a volume $V=88.78 \mathrm{~m}^{3}$ each and a 
total interior surface $S=121.15 \mathrm{~m}^{2}$ each. The Schroeder frequency $f_{s}$ of the receiving room is approximately $f_{s}=c^{3 / 2} \sqrt{T_{60} \log _{10}(e) / 4 V}=350 \mathrm{~Hz}$, assuming a reverberation time of $2.5\left[\mathrm{~s}\right.$ ] (using the averaged $T_{20}$, see also Figure 8(a)) and a propagation speed of sound $c$ of $340[\mathrm{~m} / \mathrm{s}]$. A light-weight double wall panel was mounted in the opening between both rooms, with dimensions $3.27 \times 2.98 \mathrm{~m}^{2}$. The sound field in the sending room is generated by two sound sources emitting random noise. Its response was measured at $N=145$ different measurement positions (using two interleaved meshes of 8x8 and 9x9), as shown in Figure 2 .

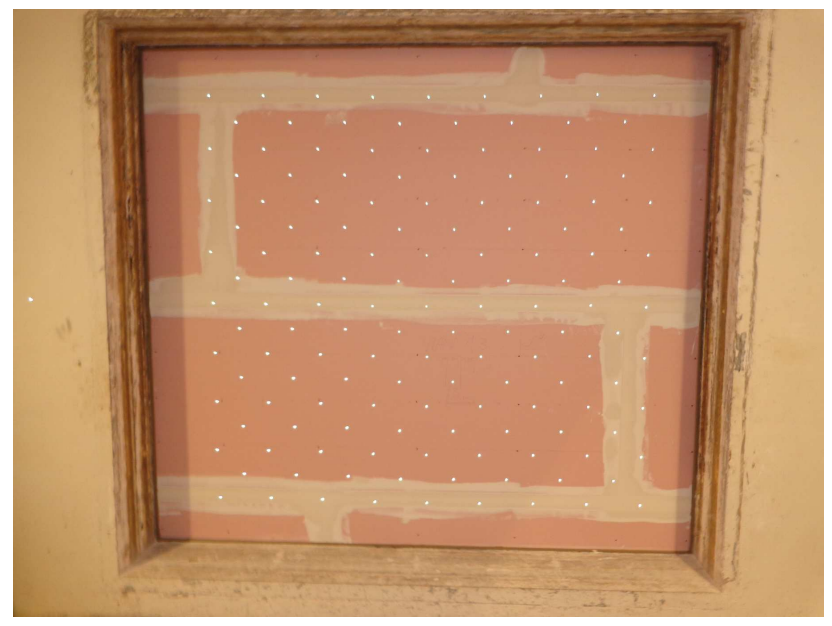

Figure 2: Double wall mounted in transmission test facility (as illustrated in Figure 1). Strips of retro-reflecting material (145 in total) are visible to enhance the optical reflection on a grid of locations on the wall, as required for the scanning laser vibrometry measurements.

Given our ambition to measure the complex (amplitude and phase) vibrational behavior of a structure subjected to a reverberant acoustic field, rather than just estimating rough amplitude levels, special care needed to be taken to acquire and process the signals. Given the diffuse character of the sound fields in both rooms, the vibration of the building element has very complex, random looking characteristics in time. Nevertheless, as the response can be assumed to be linear, each acquired response constitutes of the same modal responses. As a result, whilst the temporal responses have a random nature, the spatial response should be coherent. In order to obtain a reasonable estimate of the spatially coherent response of the building element, the required measurement record lengths need to be longer than the 
(mechanical or acoustical) reverberation time of the acousto-mechanical system involved (in this case: the transmission chambers, as these reverberation times are much longer as compared to the mechanical reverberation time of the panel).

Indeed, as mentioned by Piersol [2] in 1978, the analysis record lengths should be at least as long as the reverberation time to avoid serious errors in coherence measurements in a reverberant acoustic field. Obviously this also applies to the measurement of the vibrational response of a building element subjected to a reverberant acoustic field. In a publication by Jacobsen [3], it is mentioned that the sound field in a reverberant room can be considered fully coherent if the spectral bandwidth $\Delta f$ is less than $2.2 / T_{60}$, and in a later publication by Jacobsen [8] if the spectral spectral bandwidth $\Delta f$ is less than $1 / T_{60}$, where $T_{60}$ is the reverberation time of the room. This requirement corresponds to a record length $T=1 / \Delta f$ of at least equal to or larger than $T_{60}$.

The reason for this requirement is that, following Piersol [2], if there is a finite propagation time between two receivers, the resulting coherence measurement will include a bias error of the form

$$
\hat{\gamma}_{x y}^{2}(f)=\gamma_{x y}^{2}(f)[1-\tau / T]^{2}
$$

where $\hat{\gamma}_{x y}^{2}(f)$ is the measured coherence between the two signals $x$ and $y$, $\gamma_{x y}^{2}(f)$ is the true coherence, $\tau$ is the propagation time between the receivers and $T$ is the record length. Measurements in a reverberant acoustic field contain multiple reflections, with different propagation times, $\tau_{i}, i=1,2,3, \ldots$ The maximum $\tau_{i}$ that delivers a significant contribution to the measured signal is a function of the reverberation time of the room $T_{60}$. The same statement holds for the response of a mechanical structure that is excited by a reverberant field. Thus, in order to be able to estimate the true value of the coherence the record length $T$ should at least be as long as the reverberation time of the room $T_{60}$. Jacobsen $[3,8]$ draws the same conclusion in his papers.

Another way of explaining the requirement that the record length $T$ should at least be as long as the reverberation time of the room $T_{60}$ when dealing with a structure that is excited by a reverberant field is in terms of frequency response function and transmissibility measurements. In the present situation we measure the response $y_{i}$ at point $i$ whilst using a reference signal $y_{r}$ at a fixed position $r$. Both signals are the response due to a reverberant acoustic field. If we denote the acoustic excitation by $x$ we can 
write

$$
y_{i}=H_{i} x \quad y_{r}=H_{r} x
$$

where $H_{i}$ and $H_{r}$ are the transfer functions between an acoustic source at a specific position in the (reverberant) sending room and the vibration of the plate at points $i$ and $r$, respectively. We are interested in the estimation of the transmissibility $y_{i} / y_{r}$, which is equal to $H_{i} / H_{r}$ (in practice being estimated by means of auto- and cross-spectra, Eq. 9). The impulse responses $H_{i}$ and $H_{r}$ have a length that is approximately equal to $T_{60}$, which can be truncated at $t=T_{60}$ without important loss of signal quality. This again results in the requirement that $T$ needs to be larger than $T_{60}$ in order to be able to obtain a correct estimate of the transmissibility $y_{i} / y_{r}$, and to obtain an unbiased estimate of the coherence.

For the experimental determination of the coherent spatial response of the building element, we made use of a laser Doppler vibrometer (LDV), a Polytec OFV-505 sensor head and a Polytec OFV-5000 controller. The phase information in the signals was retained by synchronously acquiring the reference signal of an accelerometer (Bruel\&Kjaer type 4343 amplified with a Bruel\&Kjaer charge amplifier type 2635) placed on the building element approximately $20 \mathrm{~cm}$ from the bottom edge of the send-side-panel.

The coherent, structural response of the building element, including the phase relations between the measurement points, was estimated by means of the multi-pass estimator [4].

The quality of the determined low-frequency response of the building element to the reverberant sound field was assessed for different record lengths. In practice this was done by recording, for each measurement position, the two channels (the scanning laser vibrometer signal and fixed acceleration signal) in time-domain during 100 seconds using a sampling frequency of 20000 $\mathrm{Hz}$, which was downsampled to $1000 \mathrm{~Hz}$. Thus the practically imposed maximum total measurement time $T_{\max }$ equals 100 seconds. The effect of varying record length $T$ was assessed by considering parts (sub-records) of that total measurement record.

As a first step, the auto- and cross-spectra for all 145 measurement positions were estimated using a considered record length of 20 seconds (resulting in a frequency resolution of $0.05 \mathrm{~Hz}$ ), taking 8 averages with $50 \%$ overlap (thus exploiting the full data record of $100[\mathrm{~s}]$ ). The auto-spectra are shown in Figure 3. The auto-spectra of the (fixed) reference signal, as shown in Figure 3(a), is rather noisy, as expected. Using the multi-pass approach 


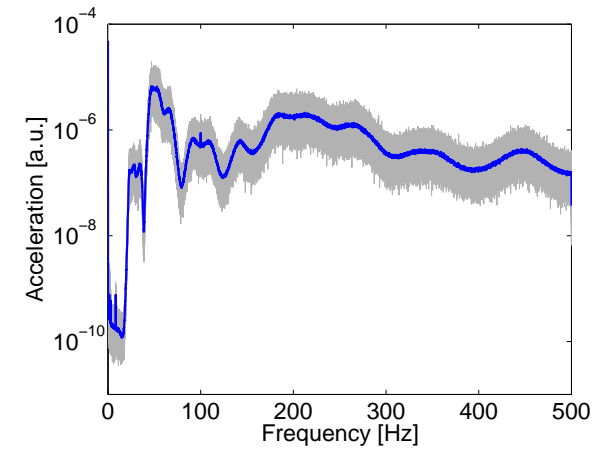

(a) Reference accelerometer autospectra $S_{r r}$ (in grey) and auto-spectra $\left\langle S_{r r}\right\rangle_{N}$, with $N=145$ (in blue).

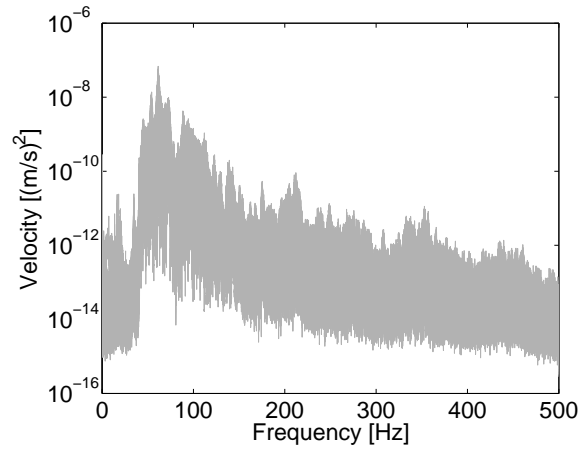

(b) Laser vibrometer auto-spectra $S_{i i}$.

Figure 3: Auto-spectra of reference accelerometer (fixed position) and laser vibrometer at 145 different measurement positions at receiving side, using a record length $T=20[\mathrm{~s}]$.

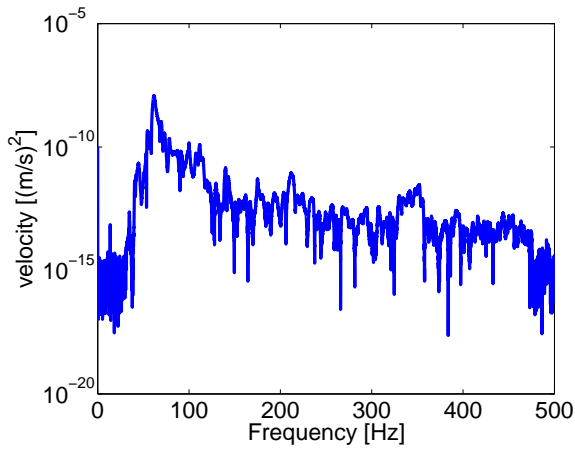

(a) Velocity auto-spectrum.

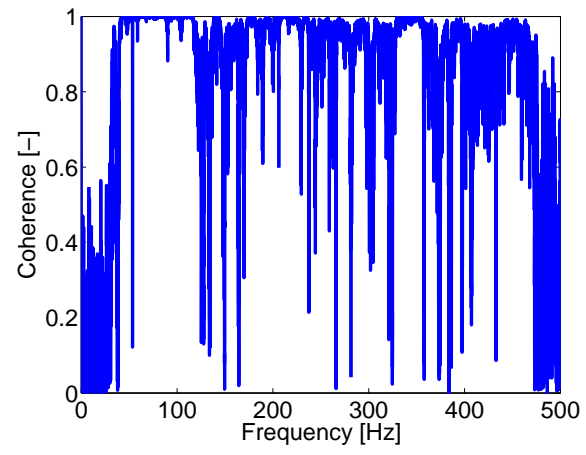

(b) Coherence.

Figure 4: Example of the multi-pass estimator for the velocity at a single point (point number 1 , at the left bottom of the building element), using a record length $T=20[\mathrm{~s}]$.

the auto-spectrum of the reference signal is determined by averaging the $N$ estimates of the auto-spectrum of the reference signal, yielding $\left\langle S_{r r}\right\rangle_{N}$, with $N=145$. This led to a substantial quality improvement (in theory a factor $\sqrt{N},[4])$ of $S_{r r}$ and consequently also of $H_{r i}$.

The final step in the data processing was to determine the individual frequency response functions for each measurement point relative to the reference signal, and calculate from these the respective response functions, by scaling with the square root estimate of $\left\langle S_{r r}\right\rangle_{N}$, using Equation (11). The 
resulting velocity spectrum $X_{i}$ at an example point and the corresponding ordinary coherence (as defined in Equation (3)) are shown in Figure 4. The ordinary coherence at this example point, as well as at other points, is relatively high in the frequency range between 40 and $115 \mathrm{~Hz}$. Below $40 \mathrm{~Hz}$, the response of the used loudspeakers was weak. Above $115 \mathrm{~Hz}$, the double wall insulated rather well, which, combined with the fact that the laser vibrometer measures velocity (and not acceleration), led to lower signal levels resulting in lower coherences.

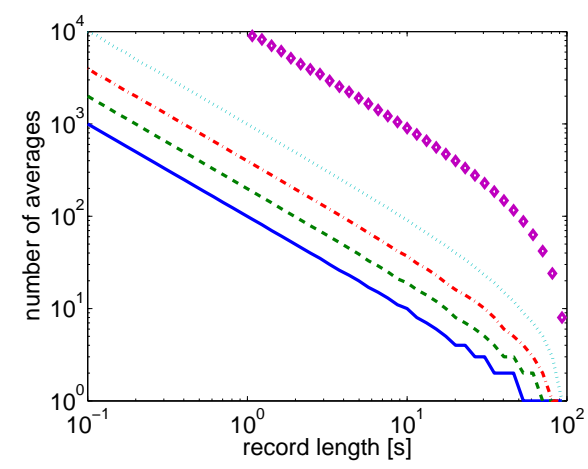

Figure 5: Dependence of the number of averages and overlap ratio, for a fixed total measurement time $T_{\max }$ of 100 seconds. Solid line: $0 \%$ overlap, dashed line: $50 \%$ overlap, dash-dotted line: $75 \%$ overlap, dotted line: $90 \%$ overlap, diamonds: $99 \%$ overlap.

We refer to Figure 5 for the relationship between the record length $T$ and the number of averages $M$ using different overlap ratios. From this figure it can be seen that the number of averages $M$ increase significantly with increasing overlap ratio's, which would appear to be interesting from a data processing point of view to increase the quality of the estimators in a cheap manner. However, it is shown in Appendix A that for randomly (Gausian) generated signals, using a Hanning window, an overlap greater than $50 \%$ does not decrease the variance of spectral estimators further, whilst Bendat's $1 / \sqrt{M}$-rule would suggest a further reduction. For instance, in the given situation, increasing the overlap from $50 \%$ to $99 \%$ would increase the number of averages from 8 to 400 (using a record length of 20 [s]; see Figure 5 ) and thus would the variance in theory be reduced by a factor $\sqrt{400 / 8}=7$. However, this predicted reduction of the variance is artificial. For that reason, in the remainder of this paper an overlap ratio of $50 \%$ is used, to ensure that the estimates for $\epsilon_{H_{r i}}^{\text {global }}$ as given in Eq. (6) will be valid.

The global coherence, as defined by Eq. 5, is depicted in Figure 6 as 


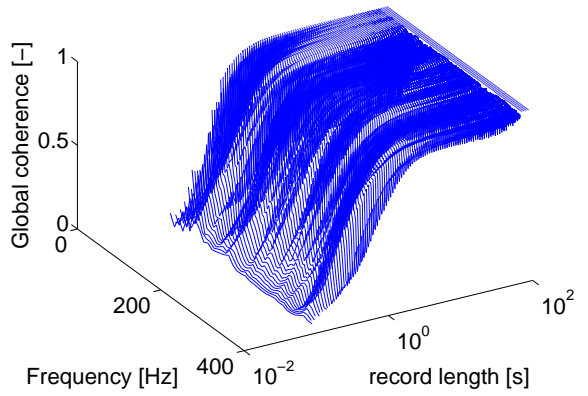

(a) $3 \mathrm{D}$ view.

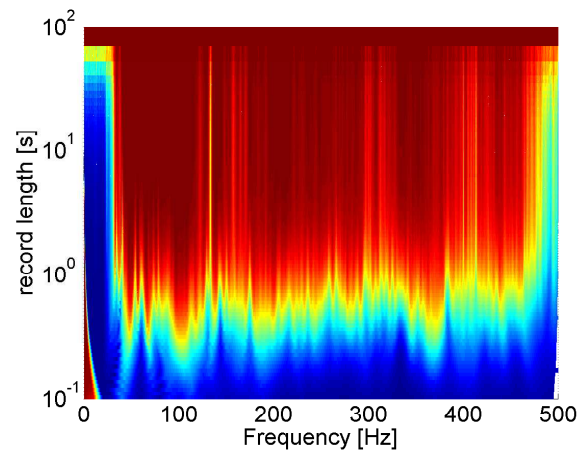

(b) Color coded top view.

Figure 6: Global coherence $\gamma^{\text {global }}$ as function of frequency and as function of the record length, using $50 \%$ overlap.

function of both frequency and record length. It can be seen that the global coherence is significantly lower than 1 for record lengths shorter than 1 or 2 seconds, which is logical as for these short acquisition times the signals are non-correlated; the measurement time is shorter as compared to the reverberation time of the rooms. For large sub-record lengths the coherence is close to 1 .

Also notice that for record lengths longer than about $70[\mathrm{~s}]$, the coherence and the global coherence is 1 for all frequencies. This is because in that case (with $50 \%$ overlap) only one averages is taken (see also Figure 6), which yields a coherence of 1 by definition. Another issue that should be mentioned here is that for small values of the number of averages $M$ the coherence value will be overestimated because not having converged to its actual value. This point is especially important because Eq. 4 is based on the actual value of the coherence, that in practice needs to be replaced by it's estimation. When the number of averages are high enough, this is allowed. But if this estimation has not converged, the coherence will be overestimated. Concluding, one should be cautious when interpreting coherence estimates and consequently also the proposed global coherence when the number of averages are low. The estimator will be reliable for values of approximately $M \geq 10$, which corresponds to a record length $T<18$ [s] for the present case $\left(T_{\max }=100\right.$ [s], 50\% overlap, see Figure 5). Fortunately, the global coherence is already rather high for record lengths smaller than $10[\mathrm{~s}]$.

The global error of the average of the response functions $\epsilon_{H_{r i}}^{g l o b a l}$ as de- 
fined by Eq. 6 is depicted in Figure 7 as function of both frequency and record length. However, the same aspect as mentioned above also applies here. When the number of averages $M$ is small the coherence values will be overestimated. The overestimation of the (global) coherence will lead to an underestimation of the uncertainty parameter as proposed by Eq. 6. Thus, the results shown in Figure 7 should be interpreted with some caution for record lengths larger than $T=18[\mathrm{~s}]$ for the present case, assuming that at least $M \geq 10$ averages are required.

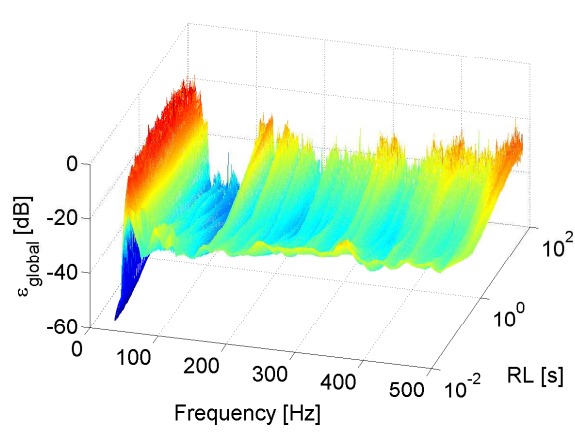

(a) $3 \mathrm{D}$ view.

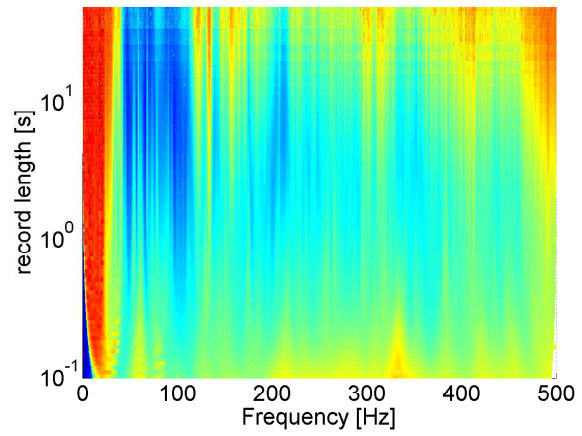

(b) Top view.

Figure 7: Global $\epsilon_{H_{r i}}^{\text {global }}$ as function of frequency and as function of the record length, using $50 \%$ overlap.

In order to enable a more global view on these results for all frequencies simultaneously, the $\epsilon^{\text {global }}$ was normalized to 1 for each frequency line individually (Figure $8(\mathrm{~b})$ ). The reverberation time $\left(T_{10}, T_{20}, T_{30}\right.$ and $\left.E D T\right)$ of the receiving room (Figure $8(\mathrm{a})$ ) was determined from the measured room impulse responses (ISO 3282) using Dirac $($ ) software with exponential sweeps as testing signal.

The optimum record length, as indicated by the minimum of the normalized $\epsilon^{\text {global }}$, is of the same order of magnitude as the reverberation time of the transmission room (Figure $8(\mathrm{~b})$ ). The reverberation time of the transmission room is in this case determining the minimum record length, as the mechanical reverberation time of the building element is much lower.

\section{Conclusions}

A global coherence parameter and a global estimate parameter for the statistical uncertainty of the average frequency response function of a multi- 


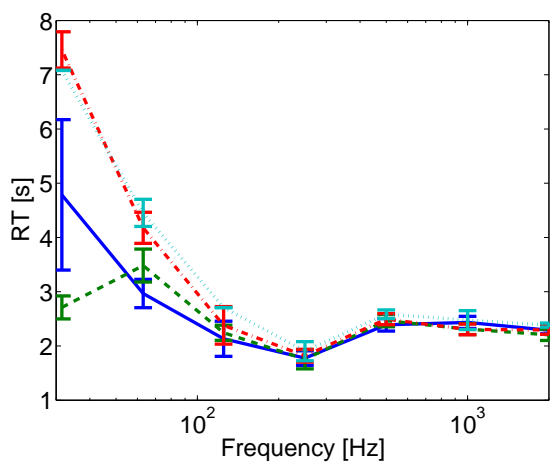

(a) Reverberation time of the receiving room, EDT (green dashed curve), $T_{10}$ (blue full curve), $T_{20}$ (red dash-dot curve), $T_{30}$ (light green dotted curve).

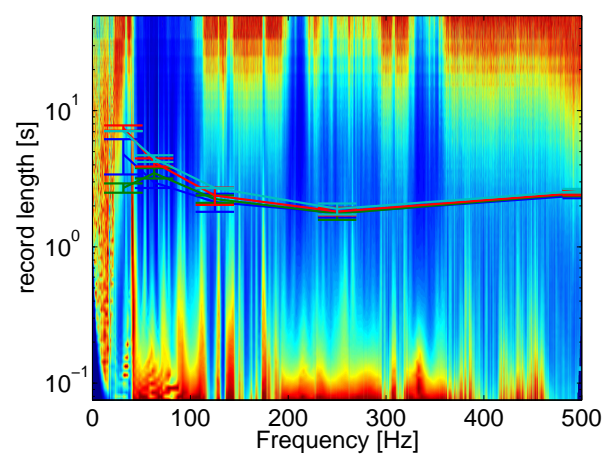

(b) Global $\epsilon_{H_{r i}}^{\text {global }}$ normalized and RT.

Figure 8: Measured reverberation time of the transmission test facility room and global $\epsilon_{H_{r i}}^{\text {global }}$ normalized for each frequency line separately, using $50 \%$ overlap, as function of frequency and as function of the record length.

channel structural-acoustic system is proposed. The proposed parameters are applied in an experimental test to assess the global uncertainty of the measured frequency response functions, and it is shown that the minimum record length needs to be larger than the reverberation time of the structuralacoustic system to avoid serious errors in the frequency response function estimate. In addition an optimal choice between the record length and the number of averages, for a fixed maximum measurement time, is determined based upon the suggested global parameters.

\section{Acknowledgements}

N.B. Roozen acknowledges support of the European Commission, Marie Curie Grant, FP7-PEOPLE-2011-IEF, Grant Agreement Number PIEF-GA2011-298278, Project acronym PAM, Project title "the Physics of Acoustic Materials". Ludovic Labelle is acknowledged for carrying out the measurements on the double wall building element. 


\section{Appendix A.}

The aim of this appendix is to show that the usable amount of overlap is limited in practice. Exceeding a certain overlap ratio will not give better estimates.

It is known that the variance of the auto-spectrum of a gaussian signal averaged over $M$ time windows is proportional to $1 / M$ [4]. Considering a signal $x$ with a rms value equal to $\bar{x}$, the variance of the double sided autospectrum $S_{x x}$ is given by (cf. [9]) :

$$
\mathbb{V}\left(\left\langle S_{x x}\right\rangle\right)=\frac{4 \bar{x}^{4}}{M n^{2}}
$$

where $n$ is the number of points of the FFT.

To illustrate the effect of overlap on the variance of the auto-spectrum of a signal, we consider a numerically generated signal with a normal distribution. The digital signal has a length of 600000 . The Fourier transform is determined for this signal with a length $n=100000$. Without overlap $M=6$ averages could be taken, whilst with $50 \%$ overlap $M=11$ averages could be take and with $99 \%$ overlap $M=500$ averages.

According to the theory above, the variance should be inversely proportional to the number of averages taken. If we estimate the variance of the auto-spectrum, which resulted from the averaging process by calculating the variance over the $n$ points of the averaged FFT-result, it corresponds well to the theoretical curve up to a certain level of the overlap ratio. But for the higher overlap ratios the theoretical curve does not correspond to the actual variance, the latter exhibiting a kind of saturation, as shown in Figure A.9. When using a Hanning window, the theoretical curve corresponds to the actual variance curve reasonably well up to about 50\% (see Figure 9(a)). Plotting the variance as function of the number of averages, obviously gives a straight line on a log-log scale for the theoretical curve (see Figure 9(c)).

It's interesting to see that the cross-over depends upon the window type being used. For instance, although a rectangular window is not recommended for stochastic signals, the theoretical curve follows the actual variance curve up to about $20 \%$ when such a window is used (see Figure $9(\mathrm{~b})$ and $9(\mathrm{~d})$ ). 


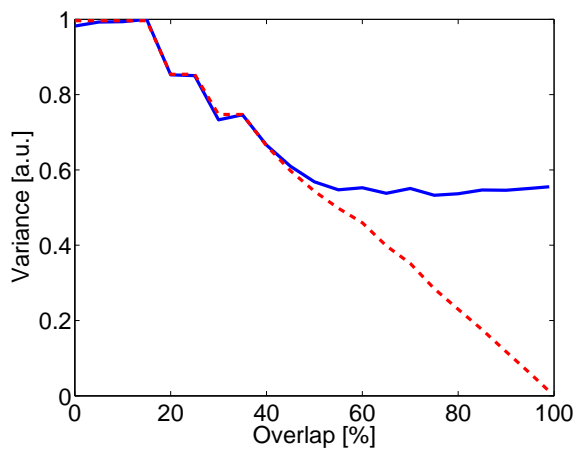

(a) Variance versus overlap ratio, using a Hanning window.

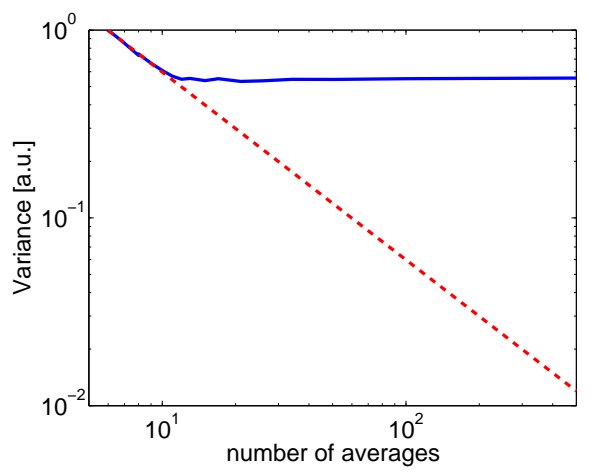

(c) Variance versus number of averages, using a Hanning window.

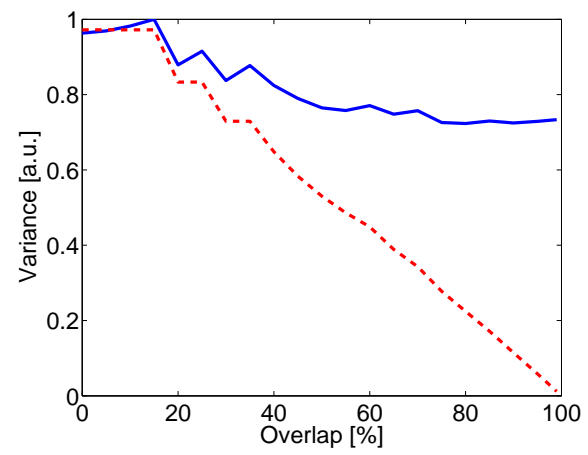

(b) Variance versus overlap ratio, using a uniform window.

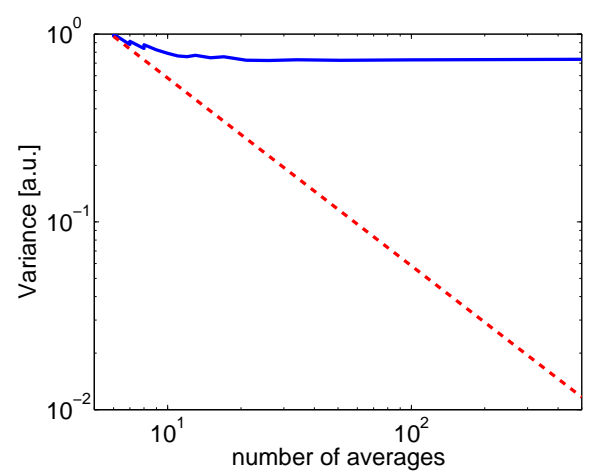

(d) Variance versus number of averages, using a uniform window.

Figure A.9: Actual variance (solid blue curve) and theoretically predicted variance (dashed red curve) for a numerically generated signal with a normal distribution, total signal length 600000 , FFT record length $n=100000$.

\section{References}

[1] N.B. Roozen, D. Pelegrín-Garcíal, M. Rychtáriková, and C. Glorieux, Determination of radiated sound power of building structures by means of scanning laser Doppler vibrometry, submitted to Acta Acoustica united with Acustica, 2014.

[2] A.G. Piersol, Use of coherence and phase data between two receivers in evaluation of noise environments, Journal of Sound and Vibration 56(2), 215-228, 1978. 
[3] F. Jacobsen and T.G. Nielsen, Spatial correlation and coherence in a reverberant sound field, Journal of Sound and Vibration 118(1), pp 175$180,1987$.

[4] J.S. Bendat, A.G. Piersol, Engineering Applications of Correlation and Spectral Analysis, Wiley-Interscience, NewYork, 1980.

[5] Alan V. Oppenheim, Ronald W. Schafer, Discrete-Time Signal Processing, Prentice Hall, 2010.

[6] R.B. Randall, Frequency analysis, Bruel\&Kjaer, Denmark, 1987.

[7] Q. Leclere, Multi-channel spectral analysis of multi-pass acquisition measurements, Mechanical Systems and Signal Processing 23 (2009) 1415-1422, doi:10.1016/j.ymssp.2008.12.002.

[8] F. Jacobsen and T. Roisina, The coherence of reverberant sound fields, J. Acoust. Soc. Am. 108 (1), July 2000.

[9] Q. Leclère, N.B. Roozen, C. Sandier, On the use of the $H_{s}$ estimator for the experimental assessment of transmissibility matrices, Mechanical Systems and Signal Processing 43 (2014), 237245. 